\title{
Editorial
}

\section{Failure Mechanisms and Damage Modelling for Advanced Materials}

\author{
Francesco Caputo, ${ }^{1}$ Carlos Navarro, ${ }^{2}$ and Paulo M. S. T. de Castro ${ }^{3}$ \\ ${ }^{1}$ Department of Industrial and Information Engineering, University of Campania "Luigi Vanvitelli", Via Roma 29, 81031 Aversa, Italy \\ ${ }^{2}$ University of Seville, Camino de los Descubrimientos $s / n$, Sevilla, Spain \\ ${ }^{3}$ FEUP, University of Porto, Rua Dr. Roberto Frias $s / n$, Porto, Portugal
}

Correspondence should be addressed to Francesco Caputo; francesco.caputo@unina2.it

Received 25 September 2017; Accepted 26 September 2017; Published 16 November 2017

Copyright (C) 2017 Francesco Caputo et al. This is an open access article distributed under the Creative Commons Attribution License, which permits unrestricted use, distribution, and reproduction in any medium, provided the original work is properly cited.

Advanced materials are widely used in several engineering applications, involving primary and secondary structures, especially where high specific strength plays a critical factor for designers. Advanced materials applications can be found in both military and civilian fields, such as aerospace, automotive, and building, and there is no shortage of applications in medical and sport equipment. However, despite their great advantages, their application is limited by many critical aspects, such as the fact that they are prone to different typologies of defects and damage respect to conventional materials. Many of such defects and damage can be very critical for structures, because they may be invisible and cause a significant decrease of the residual strength. Developing further knowhow about understanding failure mechanisms in advanced materials, due to phenomena such as impacts, fatigue, creep, and stress corrosion, is mandatory to build up a safe and efficient structure. For such reasons, before employing new materials in structures, several tests are usually carried out. Static, corrosion, impact, fatigue, high strain rate, and several other tests are usually carried out at both coupon and full scale component in order to understand the behaviour and to increase the structural performance. As a matter of the fact, the knowledge about the onset and evolution failure mechanisms in advanced materials is still not enough to face properly the design phase. Hence, during the design current practice, in order to fill the knowledge gap about the ability in predicting the failures, structural components are usually oversized. Such design strategy allows the structure to tolerate damage so its structural health is not compromised under the real loading conditions, according to a damage tolerance design philosophy. As a result, the scientific community is aimed to develop analytical, empirical, and semiempirical models able to predict the failure mechanisms under different loading conditions and nondestructive examinations, inspections, and evaluations which are widely performed to achieve all possible information about the failure mechanisms in order to develop predicting models. Achieving detailed information about such mechanisms is a necessary condition to design a safe and an efficient structure.

In more detail, the state of the art is full of atomistic models related to several types of failures, efficient for specific test cases. However, failure prediction knowledge at macro-scale lever is still poor. Empirical analyses and analytical models are often developed and used to describe failure mechanism at micro-scale level. The challenge is to transfer such detailed information to higher scales in order to reproduce the structural behaviour of a real component. Different tools are, in fact, used to achieve constitutive damage law at micro-scale level to implement inside macro-scale models able to predict the structural behaviour of a specific structure. In fact, thanks to the current computational power, multiscale numerical models are developed in order to simulate the behaviour of components, assemblies, and whole structures under both real and critical loading conditions, before proceeding to the manufacturing.

The present special issue contains original research and review articles that seek to address modelling and optimizing structural behaviour and damage of advanced materials. Among the areas emphasized in the special issue are case histories; sample calculations of practical 
design problems; material characterization procedures; failure mechanisms assessment of critical components; stochastic modelling; propagation mechanisms; effect of microstructure and defects on fatigue behaviour; life prediction and remaining useful life estimation. The papers submitted by the authors have been subjected to the normal journal peerreview process. Special topics include but are not limited to continuum mechanics, crack propagation, criteria for fatigue and fracture, multiphysics damage modelling and analysis, effect of process and design parameters on fatigue resistance, life prediction and remaining useful life estimation, scale effect and structural failure, and ageing.

Francesco Caputo

Carlos Navarro

Paulo M. S. T. de Castro 

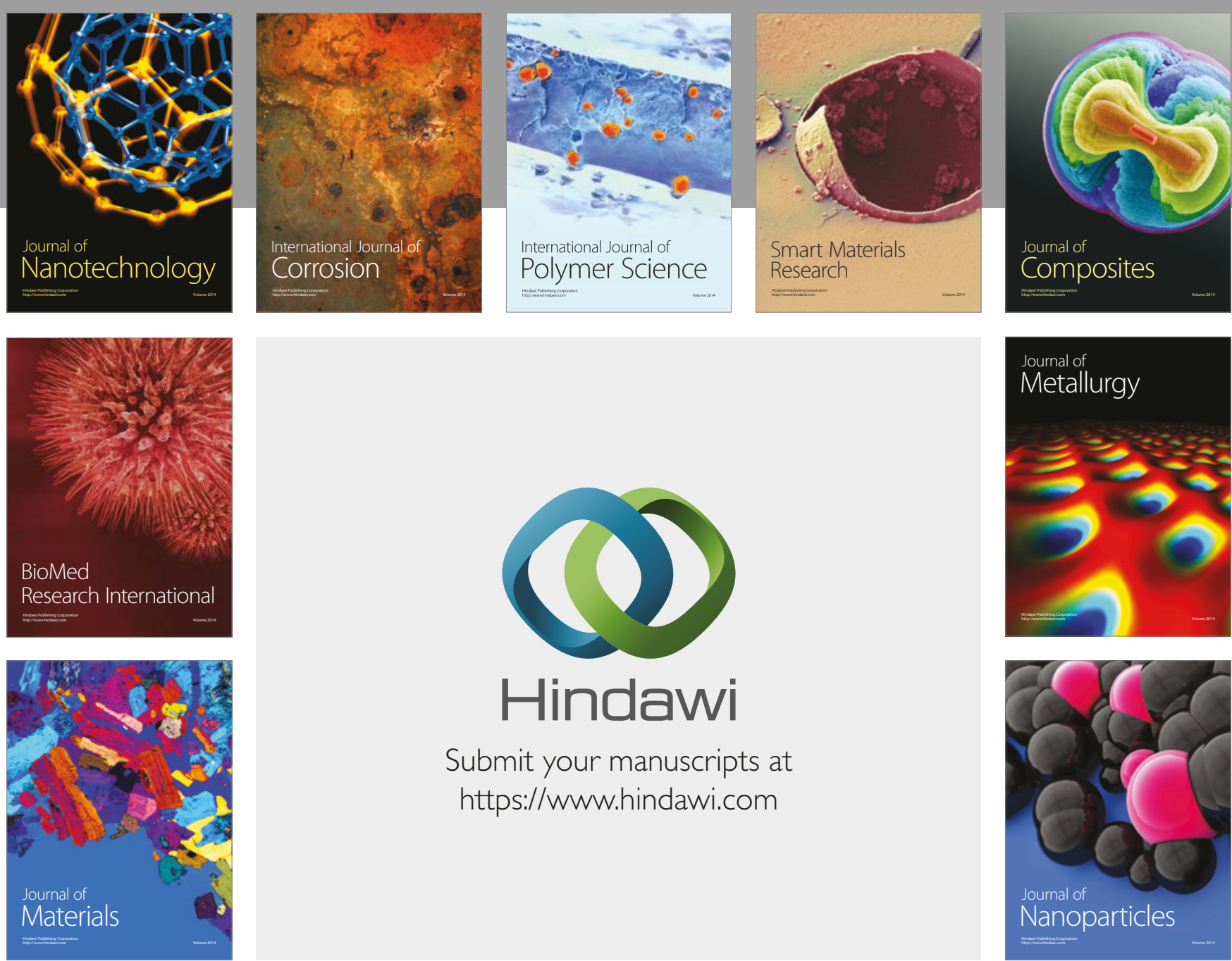

\section{Hindawi}

Submit your manuscripts at

https://www.hindawi.com
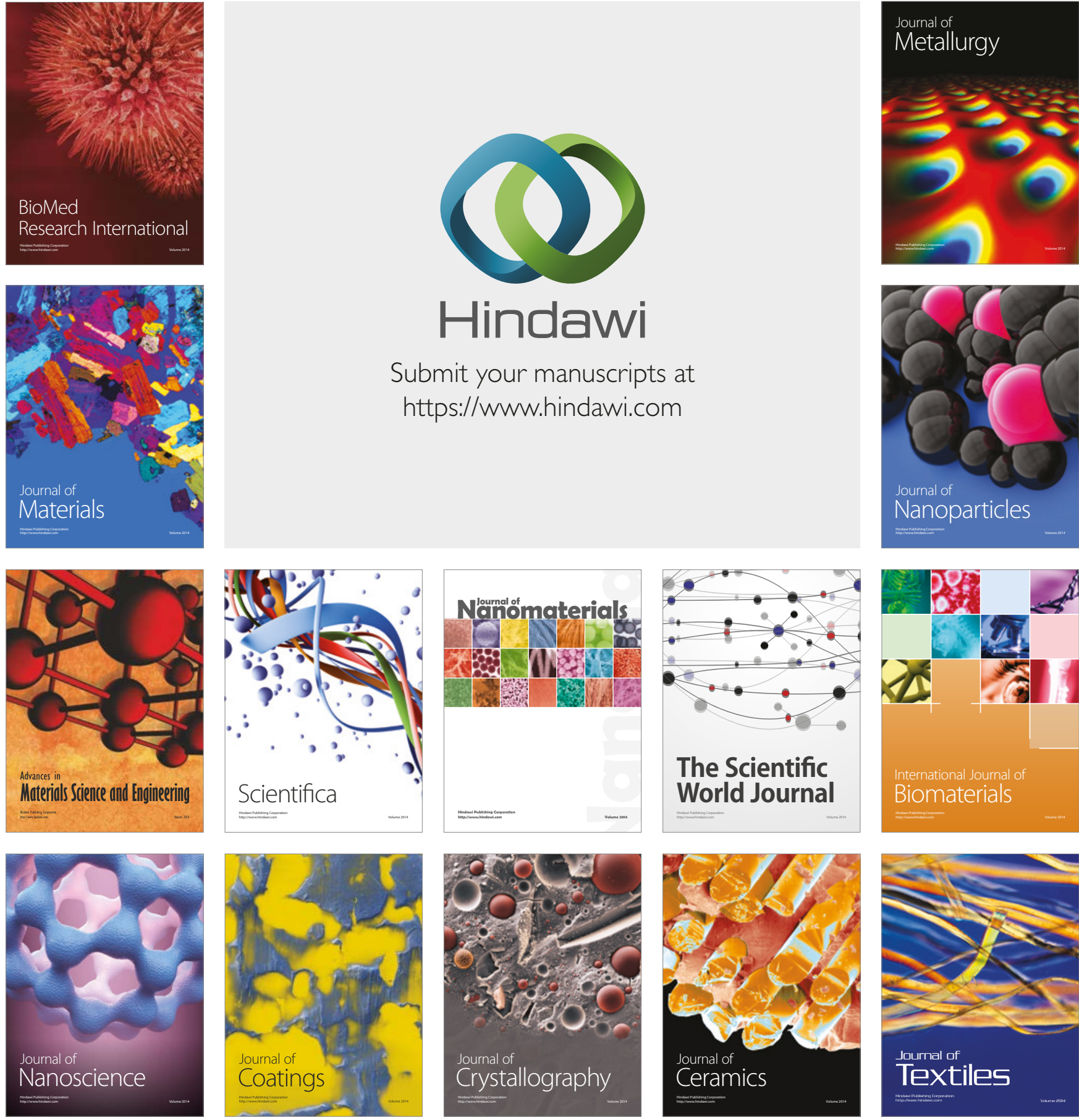



The Scientific World Journal
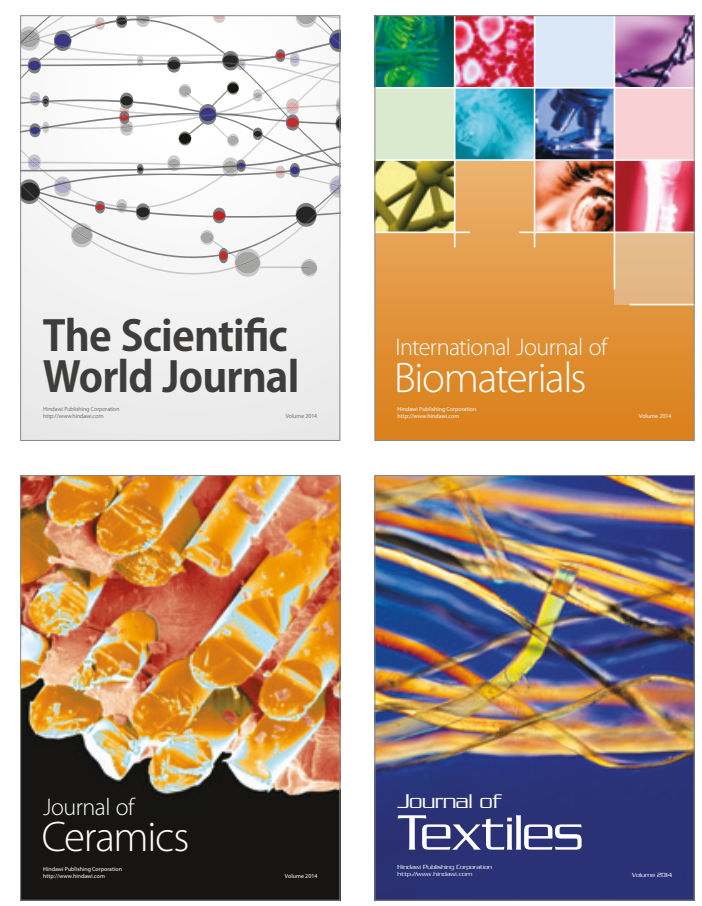